\title{
Attentional bias modification for addictive behaviors: clinical implications
}

\author{
W. Miles Cox, ${ }^{1 *}$ Javad S. Fadardi, ${ }^{1,2}$ James M. Intriligator, ${ }^{\prime}$ and Eric Klinger ${ }^{3}$
}

\footnotetext{
${ }^{1}$ School of Psychology, Bangor University, Bangor, United Kingdom

${ }^{2}$ Faculty of Education and Psychology, Ferdowsi University of Mashhad, Mashhad, Iran

${ }^{3}$ Division of Social Sciences, University of Minnesota, Morris, USA
}

When a person has a goal of drinking alcohol or using another addictive substance, the person appears to be automatically distracted by stimuli related to the goal. Because the attentional bias might propel the person to use the substance, an intervention might help modify it. In this article, we discuss techniques that have been developed to help people overcome their attentional bias for alcohol, smoking-related stimuli, drugs, or unhealthy food. We also discuss how these techniques are being adapted for use on mobile devices. The latter would allow people with an addictive behavior to use the attentional training in privacy and as frequently as needed. The attentional training techniques discussed here appear to have several advantages. They are inexpensive, can be fun to use, and have flexibility in when, where, and how often they are used. The evidence so far also suggests that they are effective.

Received 17 August 2013; Accepted 21 January 2014; First published online 19 March 2014

Key words: Addiction, attentional bias, mobile device, retraining, stroop, visual probe.

\section{Introduction}

Addictive behavior sequences are goal-directed., ${ }^{1,2}$ Whether a person drinks alcohol excessively, abuses drugs, or eats food in an uncontrolled manner, the person is aiming to achieve a goal by engaging in the behavior. The goal, for instance, might be to reduce negative things (eg, depression, anxiety, or boredom) or to enhance positive things (eg, conviviality, happiness, or courage). As with any kind of motivated behavior, when people engage in addictive behaviors, they seem to notice things in their environment that are related to achieving the goal. ${ }^{2}$ Consider, for instance, a person who drinks a lot of alcohol. Various alcohol-related objects in the environment might attract the person's attention, such as an advertisement for an alcoholic beverage, a 6-pack of beer in the refrigerator, or the aisle in a supermarket where wine is displayed.

\section{Goal-Directed or Automatic?}

Some neuroscientists would disagree that addictive behaviors are goal-directed. They view addicted individuals

\footnotetext{
*Address for correspondence: W. Miles Cox, School of Psychology, Bangor University, Bangor, Gwynedd LL57 2AS, UK.

(Emailm.cox@bangor.ac.uk)
}

as having lost control over their use of the substance to which they are addicted. The use is said to be compulsive and "automatic," ie, automatized or, to use an even more apt term, integrated ${ }^{3}$-rather than goal-directed-despite the negative consequences of the use. ${ }^{4}$ This view rests on observations that, in some cases, addicted individuals' executive control has been compromised due to brain changes that have resulted from the use. ${ }^{5}$ However, opposing automatization to goal-directedness creates a false dichotomy. Automatization means the integration of initially separate action units into a longer, relatively seamless action stream that requires little conscious control to unfold, as in skilled playing of a musical instrument, driving a car in traffic, or fielding a baseball. Indeed, bringing the components of an automatized action into consciousness tends to disrupt its flow. However, it is important to realize that automatization does not create an inflexible automaton. Even automatized action sequences are subject to modification depending on circumstances. The guitarist may change specific fingering to accommodate a suddenly broken string, the driver can adjust to the need to take a detour, and the fielder's movements will vary according to the wind, other players, or an unexpected bounce-all in the service of attaining their respective goals. The automatized action sequences remain aimed at these goals and remain under 
conscious control. A distinguishing feature of goaldirectedness-indeed, one of its operational definitionshas long been considered to be the changing tactics in the face of obstacles, as was first strongly argued by Wolfgang Köhler. ${ }^{6}$ At that time, this flexibility was labeled Umweg (detour) behavior to describe new responses that circumvent obstacles to goal-attainment. Detour behavior certainly describes the actions of addicts who face obstacles to obtaining their substances, as for instance shown in the wily actions of addicted nurses in stealing substances from patients and hospital stores. ${ }^{7}$ However addicted, their actions are not automatic in any uncontrolled sense-but, they do remain clearly directed at achieving their goals.

We agree, as do others, ${ }^{8}$ that decisions about drinking alcohol or using other substances can be highly automatized, with substance users often being unaware of the factors that influence their decisions. ${ }^{9}$ Nevertheless, the substance use itself is a voluntary act. Even if parts of a behavioral sequence become automatized, substance use remains goal-directed. The evidence suggests only that the behavior (drug seeking) can become repetitive. When we seek something, we are seeking a target (ie, a goal), regardless of whether the behavior is under selective, conscious control or whether it involves an automatized/habitual chain of behaviors that are triggered by internal or external cues.

Addiction, of course, represents a major shift in the relative incentive values of an individual's goals, both substance-related and other. In accordance with Expectancy X Value decision theory, ${ }^{11}$ that shift changes decisions and impedes a person's ability to stop striving for the target of the addiction. In other words, the goals that directing behavior change in potency, and with them the direction of decision-making changes, but the behavior remains goal-directed.

Others also contend that an addictive behavior is controlled by voluntary choices rather than being a pharmacologically controlled act. ${ }^{12-14}$ This view is consistent with the observation that even severely addicted individuals undergo natural remission, presumably when they reach the decision that the benefits of changing outweigh the costs of the addiction. ${ }^{15}$ Still others have shown that rewards and punishments can be used to dramatically change addictive behaviors, as, for example, when contingency management treatments are used. ${ }^{16}$

\section{Relevance of Attentional Bias for Addictive Behaviors}

The attentional bias for alcohol-related (or other addiction-related) stimuli apparently is more than an interesting epiphenomenon. It appears to have a real effect on the addicted person's thoughts and actions. ${ }^{17}$ For instance, when the person sees a 6-pack of beer, he or she might start thinking about how good the beer would taste and how relaxing drinking it would be. Such thoughts, in turn, might well culminate in the act of drinking, even though the person might earlier have resolved not to drink. Thus, addiction-related attentional bias seems to be an important mechanism undergirding addictive behaviors. We need, therefore, to understand its parameters and the variables that affect it. We also need ways to accurately measure it and techniques for overcoming it.

\section{Measuring Addiction-Related Attentional Bias}

Several laboratory paradigms have been developed for measuring addiction-related attentional bias, including the visual probe, ${ }^{18,19}$ flicker, ${ }^{19,20}$ dual-task, ${ }^{21}$ Stroop $^{17}$ paradigms, and eye-tracking. ${ }^{22}$ Of these, the addiction Stroop test, which is based on the classic Stroop colornaming test, has been most widely used. The stimuli used in the addiction Stroop test belong to 1 of 2 categoriesaddiction-related or emotionally neutral-and each stimulus usually appears in 1 of 4 colors: red, yellow, blue, or green. In the computerized version of the test, 1 stimulus appears on the screen at a time, and the participant's task is to ignore the meaning of the word and only name the color in which it is written as quickly and accurately as possible. When the addiction-related words are emotionally significant (eg, goal-related) for an individual, his or her attention seems to be automatically drawn to the meaning of the words, so that the time taken to name the word color is slower than when the words are emotionally neutral. From the results, an interference score is calculated for each participant (the person's mean reaction time to the addiction-related stimuli minus his or her mean reaction time to the neutral stimuli).

The literature on the addiction-related attentional bias as measured by the Stroop and other tasks is extensive, and it has been reviewed several times. ${ }^{17,23,24}$ In the case of alcohol consumption, some of the salient conclusions from these reviews are as follows:

1. The magnitude of the attentional bias is generally proportional to the amount of alcohol that people habitually consume (eg, dependent drinkers $>$ heavy drinkers $>$ moderate drinkers $>$ nondrinkers) ${ }^{17}$

2. Attentional bias and subjective craving affect each other, and this reciprocal process is likely to result in use of the substance. ${ }^{23,24}$

3. Attentional bias predicts later substance use (eg, post-treatment), although the evidence for this conclusion is not entirely consistent. ${ }^{10}$

It should be emphasized, however, that such results are based on group means (eg, the mean of heavy drinkers compared with the mean of nondrinkers). The assessment 
of attentional bias is not precise enough for definitive statements to be made about individuals' status (eg, whether or not the person is a heavy drinker or whether an addicted drinker in treatment is likely to relapse).

\section{Objectives of This Article}

The Guest Editor of this special issue of CNS Spectrums invited us to contribute an article to introduce attentional bias as a psychological construct and its relevance for the treatment of addiction disorders. Accordingly, the purpose of this article is to (a) show the relevance that attentional bias has for addictive disorders, (b) review techniques that have been developed to train people to overcome their attentional bias for addiction-related stimuli, and (c) discuss studies that have been conducted to evaluate the attentional retraining.

Attentional retraining for addictive disorders is a relatively new area of investigation. To the best of our knowledge, only 10 studies have been published on this topic, although there are a number of other studies with promising results that not been published yet. In any case, this body of literature does not yet warrant a meta-analysis or a systematic review.

Accordingly, in this article we first discuss techniques based on the Stroop color-naming test that we developed for measuring and changing people's attentional bias in the laboratory. We then discuss attentional retraining techniques that are based on the visual probe task. Finally, we discuss ongoing work that uses hand-held mobile devices for helping people to overcome their attentional bias and, in turn, their addictive behaviors.

\section{Alcohol Attention Control Training Program}

The Alcohol Attention Control Training Program $(\mathrm{AACTP})^{25}$ is a computerized intervention for helping drinkers to overcome their automatic distraction by alcohol stimuli. This distraction is thought to be the first in a series of events that can lead to positive thoughts about drinking, craving alcohol, and then procuring alcohol and imbibing. The aim of the intervention is to help drinkers gain control over the distraction caused by alcohol stimuli. If they are able to achieve this, they should then be better able to control their drinking.

The AACTP encompasses several important components. First, using the alcohol Stroop test, it measures drinkers' alcohol attentional bias, and it then informs them of the results and the meaning and consequences of their distraction. Second, it engages participants in their training with the AACTP by helping them to set realistic goals for reducing their distraction. Third, it aims to motivate participants by providing them with immediate feedback about their performance.
Like the alcohol Stroop test, AACTP training utilizes the basic principles of the classic Stroop color-naming test. There are 2 categories of stimuli: Pictures of alcoholic beverages and pictures of non-alcoholic beverages. However, both kinds of stimuli are chosen individually for each participant on the basis of his or her favorite beverages. Each stimulus has a color surround (in red, yellow, blue, or green), but the stimuli are arranged in 3 series of increasing difficulty. In the first series, on each trial an alcoholic or a non-alcoholic beverage is presented on the computer screen on a colored background. In the second series, an alcoholic or a non-alcoholic beverage has a narrow color outline rather than a colored background. In the first 2 series, the participant's task is to ignore the beverage (whether it is alcoholic or non-alcoholic) and respond to the surrounding color as quickly and accurately as possible. In the third series, an alcoholic and a non-alcoholic beverage, both of which have a narrow color outline, appear simultaneously. Here the participant is supposed to name the color of the outline of the non-alcoholic beverage while ignoring the alcoholic one. As in the alcohol Stroop test, from each of the 3 series of stimuli in the AACTP, interference scores are calculated by subtracting participants' mean reaction times to the non-alcoholic stimuli from their mean reaction times to the alcoholic stimuli. Like the 3 series of stimuli, the training itself is arranged in a hierarchy. Its difficulty varies according to which series of stimuli is presented and the time constraints placed on completing the series. Before starting the task, the participant and the person administering the training jointly agree on the time limit. It is based on what seems realistic for the participant, judging from the performance on the initial alcohol Stroop test. When the performance goal has been reached, positive feedback is given via the computer. Additionally, when each stage of the training has been completed, the participant is given feedback that includes a graph depicting the mean reaction time to the alcoholic and non-alcoholic stimuli, the alcohol interference score, and an interpretation of the scores.

The effects of AACTP training on participants' alcohol attentional bias and their alcohol consumption have been evaluated in 2 laboratory studies, and 1 study tested its effects when delivered over the Internet. In the first lab-based study, ${ }^{25}$ harmful drinkers (defined as men who drank more than 50 units of alcohol per week or women who drank more than 35 units per week) were recruited from the Bangor University Community Participant Panel and the Bangor University Intranet. Participants served as their own controls; thus, there was an initial 4-week waiting period prior to the training during which no changes in alcohol attentional bias or alcohol consumption were expected; to assess this, an assessment was given before and after the waiting period. 
Thus, during this period, participants served as a waiting-list control. ${ }^{25}$ After the 4 weeks of waiting, participants underwent 4 weeks of training, which consisted of 1 hour of AACTP training each week in our laboratory. A post-training assessment was then administered, and a follow-up assessment was administered 3 months later.

The results of the study confirmed our hypotheses. First, during the initial, 1-month waiting period, there were neither changes in participants' alcohol attentional bias nor their alcohol consumption. Second, from before to after the AACTP training, participants showed a statistically significant reduction in their alcohol attentional bias and their alcohol consumption. Third, at the 3-month follow-up, the reductions that were observed at the end of the training were maintained. These results were encouraging in that they confirmed that (a) harmful drinkers' automatic distraction by alcohol stimuli can be retrained, (b) the reduction in attentional bias is accompanied by a reduction in alcohol consumption, and (c) the improvements are sustained for at least 3 months.

The other studies that have evaluated the AACTP are as yet unpublished; nevertheless, they are worth mentioning here. In the first of these studies, ${ }^{10}$ in order to test the effects of both AACTP training and a motivational intervention, a factorial design was used to compare the individual and combined effects of the 2 interventions on alcohol consumption. Thus, unlike the first AACTP study, this study included an independent, no-treatment control group. The purpose of the motivational intervention was to identify participants' maladaptive motivational patterns and help them to change these patterns. The overarching objective was to enable participants to develop a fulfilling lifestyle that did not involve excessive use of alcohol or other drugs. In this study, both 3-month and a 6-month follow-up assessments were given. Again, the results indicated that AACTP training was effective in reducing alcohol consumption, and the reduction was sustained up to 3 months. On the other hand, although the effects of the motivational intervention were slower to occur, they lasted longer, ie, up to 6 months. There were, however, no incremental effects when the 2 interventions were combined. These results suggest that AACTP training would be effective in achieving short-term reductions in drinking, but that the effects of the motivational intervention would be more enduring.

The next study was conducted over the Internet with participants who wished to reduce their drinking. There were 4 groups of participants; they were trained with either the AACTP or 1 of 3 variants of approach-bias training, or they received sham training. At the posttest, there was a significant reduction in drinking in the AACTP group and 2 of the other 3 experimental groups.
There was also a reduction in craving, and an increase in self-efficacy and perceived control. ${ }^{26}$ Thus, the Webbased study suggests beneficial effects of the AACTP.

\section{Variants of the Attention Control Training Program}

The importance of attentional bias is not limited to alcohol abuse. For example, smokers show an attentional bias for smoking-related stimuli, ${ }^{17,27-33}$ and drug abusers show an attentional bias for drug-related stimuli. ${ }^{24,34-36}$ Moreover, the substance-related attentional bias appears to increase abusers' substance-seeking and substance use. Several researchers have also tested the effectiveness of attentional bias training in helping smokers curb their attentional bias for addiction-related stimuli. ${ }^{37,38}$ Nevertheless, even though drug-related attentional bias predicts post-treatment relapse among drug abusers, ${ }^{23,39,40}$ to our best knowledge, attentional training has not previously been developed for drug abusers.

This omission is important because drug abusers might be characteristically different from alcohol abusers. ${ }^{41,42}$ Moreover, most attentional bias research has been conducted with abusers from Western cultures. Thus, 1 question remained unanswered: To what extent can we generalize findings about alcohol attentional bias to drug abusers given that a considerable number of drug abusers live in non-Western countries?

Two unpublished studies ${ }^{43,44}$ found that Iranian heroin and opium abusers had greater attentional bias for drug-related stimuli than non-abusers. Another published study ${ }^{45}$ showed that drug-related attentional bias was inversely related to attentional bias for personally relevant goals; that is, as distraction for stimuli related to participants' personal (non-substance-related) goals increased, distractions for drug-related stimuli decreased. Studies from South Korea that used eye-tracking techniques ${ }^{46,47}$ found that smokers had an attentional bias for smokingrelated cues. Another study that combined eye tracking with functional magnetic resonance imaging (fMRI) ${ }^{48}$ concluded that the brain's attentional mechanisms in combination with its motivational and reward-related mechanisms play an important role in smoking-related cue reactivity, attentional bias, and subjective craving. Studies from China have reported similar results. ${ }^{49,50}$ These results underscore the importance of developing attentional training for drug abusers.

Encouraged by the results obtained using the AACTP with alcohol abusers discussed earlier, we developed and tested the effectiveness of the Drug Alcohol Attention Control Training Program (Drug-ACTP) using 2 samples: (a) drug abusers having methadone maintenance therapy (MMT) ${ }^{43}$ and (b) drug abusers undergoing detoxification. ${ }^{44}$ It should be noted, however, that 2 of these studies are as yet unpublished. The procedure for the Drug-ACTP is similar to that for the 
AACTP; however, the salient stimuli are drug-related rather than drug-related. These include pictures of drugs, such as heroin, opium, and pills, and drug-abuse equipment such as pipes and lighters. We first collected a large number of photographs of drug-related items, then showed them to drug-abusers in treatment and asked them to rank the pictures according to their relevance to drug abuse and their putative salience. Then, the most relevant and salient photos were selected for inclusion in the Drug-ACTP. Similarly, pictures of fruits and vegetables were assembled and shown to drug abusers to rank in terms of their popularity and desirability, and those with the highest ranks were chosen for inclusion in the program. The rationale for including fruits and vegetables was to have a healthy alternative to drugs.

In the first study, ${ }^{43}$ volunteer drug abusers in MMT received 3 45-minute training sessions with the Drug-ACTP. The control group comprised a comparable sample of drug abusers on MMT who continued receiving treatment as usual. An assessment was given at baseline, post-training, and 2 and 6 months later. It measured, among other things, attentional bias, motivational structure, ${ }^{51,52}$ temptation to use, confidence to resist using, and readiness to change. Results showed that, compared to the control group, the experimental group (a) reduced their drug-related attentional bias (Cohen $d=0.66$ ), temptations to use (Cohen $d=0.64$ ), and number of lapses (Cohen $d=0.67$ ); and (b) they increased their confidence to resist temptations and urges (Cohen $d=0.73$ ). Moreover, regardless of group membership, participants' adaptive motivation was positively correlated with success in achieving their therapeutic goals (in terms of months that they remained in the MMT program), and was negatively associated with the dose of methadone taken and number of relapses. Thus, these results are consistent with those obtained with the AACTP. Nevertheless, one might argue that drug abusers in MMT, knowing that they are aided by methadone, face minimal difficulty in managing their sobriety. It was important, therefore, to establish the effectiveness of the Drug-ACTP with drug abusers who were not taking a medication, such as methadone or buprenorphine, to counteract the effects of opium withdrawal.

The second study, ${ }^{44}$ therefore, was conducted with drug abusers in a detoxification program. They were randomly assigned (a) to receive 3 sessions of training with the Drug-ACTP or (b) to a control group that received a computerized sham intervention. Participants received the same assessment tools as in the MMT study, and they were retested post-training and 1 month and 6 months later. At 1 or more of the follow-ups, training with the Drug-ACTP, unlike the sham training, had reduced drug-related attentional bias (Cohen $d=0.77$ ), perceived stress (Cohen $d=0.90)$, temptation to use
(Cohen $d=0.70$ ), negative affect (Cohen $d=0.77$ ), and number of relapses. Moreover, at post-treatment and the 6-month follow-up, these reductions were accompanied by increased confidence to resist using drugs (Cohen $d=0.62$ ). Therefore, similar to the drug-abusers in MMT, those in detoxification benefited from the training. These findings support the clinical usefulness of the Drug-ACTP.

We believe that attentional training using the ACTP procedure could be used with many problems where decision-making (eg, what to eat) or feeling a certain way (eg, depressed, anxious) is a critical variable in maintaining the problematic behavior. We have conducted 3 studies that used the ACTP with dieting, depressed, or socially anxious individuals. These kinds of problems have communalities with alcohol and drug abuse; the most important is the person's perception of loss of control over various aspects of the problem. For example, cognitive factors (especially attentional bias ${ }^{53-56}$ ) play an important role in the eating behavior of dieters. Overweight people's eating behavior is, of course, an important determinant of their success in losing weight, and attentional training might become an important therapeutic component of dieting interventions.

Of the 3 studies using the ACTP we just referred to (with dieting, depressed, or socially anxious individuals), we discuss only the one with dieters because eating behavior, unlike the other 2 problems, can be regarded as an addictive disorder. The study had 2 goals. ${ }^{57}$ The first goal was to study the relationship between attentional bias and eating behavior in dieters who did not have a severe eating disorder (ie, anorexia nervosa or bulimia nervosa). The results showed that food-related attentional bias was greater in dieters than in people of normal body weight who were not dieting, and that participants' body-mass index was positively associated with their attentional bias for high-calorie food. Moreover, obese dieters were characterized by emotional (eating in response to negative emotions) and restrained (self-restricted food intake) eating rather than external eating. The second goal of the study was to test the effectiveness of a food-ACTP in reducing dieters' attentional bias for high-calorie food. Dieters were randomly assigned to 1 of 3 groups: active training, sham training, or a no-intervention control. An assessment was given to all groups at baseline, post-training, and a 3-month follow-up. Training with the Food-ACTP reduced dieters' food-related attentional bias and the rate at which they dropped out of a dieting program.

\section{Other Attentional Retraining Techniques}

Attentional retraining techniques for addictive behaviors have been developed using paradigms other than the Stroop task, most notably the visual-probe task. 
In the assessment version of the visual-probe task, the stimuli are 2 pictures or 2 words, presented side by side on a computer screen, 1 of which is alcohol-related and the other neutral. The stimuli then disappear; a visual probe appears in the location of 1 of the stimuli, and the participant is instructed to respond to the probe as rapidly as possible. Many studies have shown that, unlike individuals who are not abusing drugs, heavy drinkers, tobacco smokers, heroin users, and cocaine users are faster to respond to probes that replace drugrelated pictures than to probes that replace neutral pictures, suggesting that the drug-related cues capture drug abusers' attention. ${ }^{23}$

In the training version of the task, the location of the visual probes is manipulated in such a way that participants can be taught to direct their attention either toward or away from the location that the drugrelated stimulus occupied. For example, to train participants to direct their attention away from drugrelated cues, visual probes would replace nondrugrelated, control pictures with a probability close to $100 \%$. Over a series of trials, participants shift their attention away from the drug-related stimuli in order to respond rapidly to the probes.

This training technique has been used with heavy drinkers, and it has consistently affected alcohol attentional bias in the predicted direction. ${ }^{18,19,58}$ As also hypothesized, it has sometimes affected subjective ratings of craving and the amount of alcohol consumed in an experimental taste test. The technique has been used with alcohol-dependent patients, with whom it had the expected effect on attentional bias. Of greater clinical significance, one study gave multiple sessions of attentional training to alcohol-dependent inpatients. ${ }^{59}$ The intervention led to a reduction in alcohol attentional bias that generalized to novel alcohol stimuli, and multiple indices of clinical outcome (eg, clinicians' judgments of treatment success, time to relapse) were better in the intervention than in the control group.

Finally, a study of attentional bias training using the visual-probe task with cigarette smokers found that training to attend or to avoid attending to target cues increased and decreased, respectively, participants' attentional bias for smoking-related cues. ${ }^{40}$ There was also some evidence that training to attend to smoking cues increased participants' craving to smoke.

\section{Mobile Versions of Attentional Retraining}

Given the successful results of the various laboratorybased interventions discussed in this article, it is natural to wonder whether the same techniques could be brought "out of the lab" and used by individuals on mobile devices (eg, iPhones, Androids, or other smartphones). Making such training techniques available on mobile devices would have several advantages. For example, it would allow individuals who are fighting an addiction to participate in the training as and when they needed it and in a private environment. Furthermore, having the training on a mobile device would allow it to be used more frequently, and some evidence suggests that training which is "spaced" is more effective than training which is "massed." 60,61 This is related to the well-known "spacing effect" that has been extensively studied in the memory and learning literature.

Finally, depending on the specific implementation, it may also be possible to incorporate "game-like" elements into the training (eg, by using leaderboards, high scores, or other challenges) that may serve to increase trainees' engagement.

Within non-addiction domains, several cognitive and attention-related training paradigms have been developed for mobile devices. Most notably, many versions of the well-known "n-back memory task"62 have been developed for mobile devices. These have gained popularity because research suggests that practice on the task may improve fluid intelligence. ${ }^{63}$

Furthermore, the self-help mobile market has had a proliferation of mobile-training programs that are aimed at helping users overcome various forms of anxiety (including social anxiety disorder), phobias, and depression. Most of these have been built around variations of the initial visual-probe task ${ }^{64}$ that was described earlier. In the self-help and mobile versions of such tasks, the mobile user sees pairs of photographs-one neutral image and the other containing a concern-related stimulus (such as a spider [for people suffering from a spider phobia], a sad face [for depressed individuals], or a group of people [for socially anxious individuals]). Both images then disappear, and a target probe replaces 1 of the 2 images. The user is instructed to respond to the target as quickly as possible. In the measurement phase, the target replaces either the neutral image or the concern-related image with equal probability. The classic finding is that users are faster and more accurate at detecting or classifying the target when it replaces the concern-related image. This presumably occurs because the user's attention is involuntarily drawn to the concern-related stimulus. When this paradigm is used as a retraining task, the target always (or with a greater-than-chance probability) replaces the neutral stimulus. Although initially performance is poor (due to the attention-grabbing nature of the concern-related stimuli), over time attention is retrained, speed and/or accuracy of responding to the target stimulus increases, and (ideally) the attentional bias decreases. In laboratory situations, the training has been shown to lead to a reduction in the relevant anxiety, phobia, or other problem. ${ }^{65,66}$

Whereas numerous researchers have studied cognitive and anxiety-related training programs on mobile 
devices, to date, addiction-specific research has received only minimal attention. One company has made a version of the visual-probe task that is aimed at helping mobile device users retrain their attention in order to avoid a range of concern-related stimuli, including smoking-related ones. The company, Mental Mint (http://www.mentalmint.com/), uses the visual-probe paradigm described above. Although there are currently no efficacy data available for these programs, the company has managed to incorporate a variety of engagement-enhancing features (scores, tips, facts, etc) into its program.

Recently we have developed a "gamified" version of the ACTP. Unlike the standard visual-probe variants made for mobile phones, we have taken the underlying principles on which the lab-based procedure is based and incorporated them into an arcade-style game. Mobile phone users have a first-person perspective of a shopper going through a 3D supermarket. They must run down the cartoon-style supermarket aisle and collect healthy items and avoid grabbing the alcoholrelated items. Grabbing unhealthy items or missing healthy items leads to point losses and ultimately to failure to progress to higher levels. At the end of each 45-second "run," a mad dash round has pairs of items appearing, and the player must touch the non-alcohol item in the pair (very much like the original AACTP). The game is designed with numerous gamification features, including levels, high-scores, time limits, subtle increases in difficulty, sound effects, etc. To ensure a broad demographic sample, and to collect blind data, we partnered with a professional media research agency (Other Lines of Enquiry, http://www.otherlines. com) to run a field study using the new game.

In the first field study, we identified 37 (60\% male) heavy drinkers whose habitual alcohol consumption was well above the UK Department of Health's genderspecific guidelines for safe drinking. From this pool of drinkers, 32 were selected to form the experimental group and were asked to play the game for at least 12 minutes a week (at their leisure); the remaining 8 served as a waiting-list control group. We used the alcohol Stroop task to quantify participants' attentional bias before the intervention, after 2 weeks, after 4 weeks (when the study had ended), and again at 8 weeks. The testing sessions also included several drinking-related questionnaires to measure, for example, participants' alcohol consumption and their motivation to change. The first finding worth noting is the amount of time spent engaged with the game. Although participants were required to play for only 12 minutes a week, the mean time playing was approximately 30 minutes, with some individuals playing more than 1 hour each week. This suggests that the users found the game engaging and offers hope for creating an intervention-like training program that people will find intrinsically rewarding. In terms of behavioral manifestations of the training, we measured the amount of alcohol consumed on typical drinking days and heavier-thanusual days. Prior to the training, the experimental group consumed an average of 19.7 units of alcohol on typical drinking days and 24 units on heavier drinking days. After 2 weeks of playing the game, their drinking had decreased to 10.6 units on typical days and 15.7 units on heavier days. After a further 2 weeks (ie, at the end of the 4-week intervention), the levels consumed remained at this lower level (11.1 units and 15.5 units), and at the later (8-week) follow-up, after participants had stopped playing the game for 4 weeks, the units consumed actually decreased even more (to 8.6 and 12.5 units/day).

The mean total score on the Drinker Inventory of Consequences $^{67}$ also decreased in this group from 21.9 before playing the game to 16.2 after 2 weeks of playing and to 14.8 after an additional 4 weeks, and the reduction was maintained at 13.9 at the post-play, 4-week follow-up. We also found improvements in situational confidence across the testing periods (with a mean score before training of 71 , which increased to 72 at 2 weeks, to 74 at 4 weeks, and to 76 at the 4 -week follow-up). We also found improvements in an array of other behaviors related to health, work, and self-esteem. Interestingly, we also found some changes in variables that suggest that we are not seeing purely socialdesirability or pleasing-the-experimenter effects. For example, across the various sessions, participants reported liking the taste of alcohol more and enjoying their drinking more. The most optimistic interpretation is that the participants were learning to moderate and enjoy their drinking in a more responsible and less harmful way.

Initial feedback on the game suggests that it is easy to use $(70 \%$ agreed) and enjoyable to play (65\% agreed). High attrition among the control group makes it difficult to make direct comparisons between the 2 groups, but this may be another indicator that the game itself is engaging. At this point, these initial, very encouraging results are being followed up in a larger sample with a more appropriate control group.

\section{Conclusions}

As this article shows, attentional bias for substancerelated stimuli has been commonly observed among individuals with a substance-use disorder, and having a substance-related attentional bias increases the likelihood that a substance user will actually use now and in the future. ${ }^{33,68}$ Training techniques have been developed for helping substance users to overcome their attentional distraction for substance-related stimuli. These techni- 
ques have been shown to be effective in reducing both attentional bias and substance use. We think that it is important for both clinicians and substance users who are attempting to change their behavior to be aware of the role that attentional bias might play in maintaining the unwanted behavior. It is also important for them to be aware of the options that are available for overcoming the attentional bias.

We believe that attentional training should be given more weight in intervention programs for addiction disorders, because attentional bias seems to play a role in the uncontrollability of the behavior. The preliminary evidence for the effectiveness of attentional training for addictive disorders is encouraging, although rigorous clinical trials have not yet been conducted. As noted in this article, there are many advantages of computerized and mobile attentional training. The training is inexpensive and easy to administer, it can be fun to use, it can be scheduled and adjusted to meet each individual's needs, and it can be used in a person's home on his or her own electronic devices.

\section{Disclosures}

The work described in this article was supported by Economic and Social Research Council Grant RES-00023-9563 (awarded to W. Miles Cox and Emmanuel M. Pothos), Economic and Social Research Council Grant RES-000-22-0314 (awarded to W. Miles Cox and Javad Salehi Fadardi), Economic and Social Research Council Grant RES-000-23-1269 (awarded to W. Miles Cox, Javad S. Fadardi, Steven G. Hosier, and Emmanuel M. Pothos), and by several research and development contracts from the UK Department of Health and the National Health Service. Dr. Klinger does not have anything to disclose.

\section{REFERENCES:}

1. Cox WM, Klinger E. A motivational model of alcohol use: determinants of use and change. In: Cox WM, Klinger E, eds. Handbook of Motivational Counseling: Goal-Based Approaches to Assessment and Intervention with Addiction and Other Problems. Chichester, UK: Wiley-Blackwell; 2011: 131-158.

2. Klinger E, Cox WM. Motivation and the goal theory of current concerns. In: Cox WM, Klinger E, eds. Handbook of Motivational Counseling: Goal-Based Approaches to Assessment and Intervention with Addiction and Other Problems. Chichester, UK: WileyBlackwell; 2011: 1-47.

3. Klinger E. Structure and Functions of Fantasy. New York: Wiley-Interscience; 1971.

4. Belin D, Belin-Rauscent A, Murray J, Everitt B. Addiction: failure of control over maladaptive incentive habits. Curr Opin Neurobiol. $2013 ; \mathbf{2 3}(4)$ : $564-572$.

5. Everitt B, Robbins T. From the ventral to the dorsal striatum: devolving views of their roles in drug addiction. Neurosci Biobehav Rev. 2013; 37(9 Pt A): 1946-1954.
6. Köhler W. The Mentality of Apes. New York: Harcourt, Brace \& Company; 1925.

7. Stahl B. Addicted nurses keep licenses. StarTribune. 2013; 32: A31, A38-A39.

8. Tiffany ST, Conklin CA. A cognitive processing model of alcohol craving and compulsive alcohol use. Addiction. 2000; 95(Suppl 2): S145-S153.

9. Wiers RW, Cox WM, Field M, et al. The search for new ways to change implicit alcohol-related cognitions in heavy drinkers. Alcohol Clin Exp Res. 2006; 30(2): 320-331.

10. Field M, Marhe R, Franken IH. The clinical relevance of attentional bias in substance use disorders. CNS Spectr. 2013; 30: 1-6.

11. Van Eerde W, Thierry H. Vroom's expectancy models and workrelated criteria: a meta-analysis. J Appl Psychol. 1996; 81(5): 575-586.

12. Heyman G. Addiction and choice: theory and new data. Front Psychiatry. 2013; 4: 31 .

13. Heyman G. Resolving the contradictions of addiction. Behav Brain Sci. 1996; 19(4): 561-574.

14. Russell C, Davies JB. Empirical, logical and philosophical arguments against cigarette smoking as a pharmacologically compelled act. Current Psychology. 2009; 28(3): 147-168.

15. Bischof G, Rumpf H-J, John, U. Natural recovery from addiction. In: Shaffer H, LaPlante D, Nelson S, eds. APA Addiction Syndrome Handbook, Vol 2: Recovery, Prevention, and Other Issues. Washington, DC: American Psychological Association; 2012: 133-155.

16. Stitzer M, Petry N. Contingency management for treatment of substance abuse. Annu Rev Clin Psychol. 2006; 2: 411-434.

17. Cox WM, Fadardi JS, Pothos EM. The addiction-Stroop test: theoretical considerations and procedural recommendations. Psychol Bull. 2006; 132(3): 443-476.

18. Field M, Duka T, Eastwood B, et al. Experimental manipulation of attentional biases in heavy drinkers: do the effects generalise? Psychopharmacology (Berl). 2007; 192(4): 593-608.

19. Schoenmakers T, Wiers RW, Jones BT, Bruce G, Jansen AT. Attentional re-training decreases attentional bias in heavy drinkers without generalization. Addiction. 2007; 102(3): $399-405$.

20. Jones BT, Jones BC, Smith H, Copley N. A flicker paradigm for inducing change blindness reveals alcohol and cannabis information processing biases in social users. Addiction. 2003; 98(2): 235-244.

21. Waters H, Green MW. A demonstration of attentional bias, using a novel dual task paradigm, towards clinically salient material in recovering alcohol abuse patients? Psychol Med. 2003; 33(3): 491-498.

22. Friese M, Bargas-Avila J, Hofmann W, Wiers R. Here's looking at you, bud: Alcohol-related memory structures predict eye movements for social drinkers with low executive control. Social Psychological and Personality Science. 2010; 1(2): 143-151.

23. Field M, Cox WM. Attentional bias in addictive behaviors: a review of its development, causes, and consequences. Drug Alcohol Depend. 2008; 97(1-2): 1-20.

24. Field M, Munafo MR, Franken IHA. A meta-analytic investigation of the relationship between attentional bias and subjective craving in substance abuse. Psychol Bull. 2009; 135(4): 589-607.

25. Fadardi JS, Cox WM. Reversing the sequence: reducing alcohol consumption by overcoming alcohol attentional bias. Drug Alcohol Depend. 2009; 101(3): 137-145.

26. Wiers R, Houben K, Fadardi J, van Beek P, Cox WM. Alcohol approach-bias and attentional retraining for problem drinkers over the Web. Manuscript submitted for publication. 2014. 
27. Dawkins L, Powell JH, West R, Powell J, Pickering A. A doubleblind placebo controlled experimental study of nicotine: I-effects on incentive motivation. Psychopharmacology (Berl). 2006; 189(3): 355-367.

28. Dickter CL, Forestell CA. Peering through the smoke: the effect of parental smoking behavior and addiction on daily smokers' attentional bias to smoking cues. Addict Behav. 2012; 37(2): 187-192.

29. Gilbert DG, Sugai C, Zuo Y, et al. Brain indices of nicotine's effects on attentional bias to smoking and emotional pictures and to task-relevant targets. Nicotine Tob Res. 2007; 9(3): 351-363.

30. Johnsen BH, Thayer JF, Laberg JC, Asbjornsen AE. Attentional bias in active smokers, abstinent smokers, and nonsmokers. Addict Behav. 1997; 22(6): 813-817.

31. Mogg K, Bradley BP. Selective processing of smoking-related cues in smokers: manipulation of deprivation level and comparison of three measures of processing bias. J Psychopharmacol. 2002; 16(4): 385-392

32. Traylor AC, Bordnick PS, Carter BL. Using virtual reality to assess young adult smokers' attention to cues. Cyberpsychol Behav. 2009; 12(4): 373-378.

33. Waters AJ, Shiffman S, Sayette MA, et al. Attentional bias predicts outcome in smoking cessation. Health Psychol. 2003; 22(4): 378-387.

34. Hester R, Dixon V, Garavan H. A consistent attentional bias for drug-related material in active cocaine users across word and picture versions of the emotional Stroop task. Drug Alcohol Depend. 2006; 81(3): 251-257.

35. Hogarth L, Dickinson A, Duka T. Detection versus sustained attention to drug cues have dissociable roles in mediating drug seeking behavior. Exp Clin Psychopharmacol. 2009; 17(1) 21-30.

36. Rosenberg H. Clinical and laboratory assessment of the subjective experience of drug craving. Clin Psychol Rev. 2009; 29(6): 519-534.

37. Attwood AS, O’Sullivan H, Leonards U, Mackintosh B, Munafo MR Attentional bias training and cue reactivity in cigarette smokers. Addiction. 2008; 103(11): 1875-1882.

38. Field M, Duka T, Tyler E, Schoenmakers T. Attentional bias modification in tobacco smokers. Nicotine Tob Res. 2009; 11(7): 812-822.

39. Goldstein RZ, Woicik PA, Lukasik T, Maloney T, Volkow ND. Drug fluency: a potential marker for cocaine use disorders. Drug Alcohol Depend. 2007; 89(1): 97-101.

40. Marissen MAE, Franken IHA, Waters AJ, et al. Attentional bias predicts heroin relapse following treatment. Addiction. 2006; 101(9): 1306-1312.

41. Conway KP, Kane RJ, Ball SA, Poling JC, Rounsaville BJ. Personality, substance of choice, and polysubstance involvement among substance dependent patients. Drug Alcohol Depend. 2003 ; 71(1): $65-75$

42. Colpaert K, Vanderplasschen W, De Maeyer J, Broekaert E, De Fruyt F. Prevalence and determinants of personality disorders in a clinical sample of alcohol-, drug-, and dual-dependent patients. Subst Use Misuse. 2012; 47(6): 649-661.

43. Ziaee S, Fadardi JS, Cox WM. Effects of attention control training on drug abusers' drug attentional bias and recovery indices. Mashhad, Iran: Ferdowsi University of Mashhad; 2013.

44. Barerfan Z, Fadardi JS, Cox WM. Effects of attention-control training on detoxified drug abusers' attentional bias and other treatment indices. Mashhad, Iran: Ferdowsi University of Mashhad; 2013.
45. Fadardi JS, Ziaee S, Shamloo ZS. Substance use and the paradox of good and bad attentional bias. Exp Clin Psychopharmacol. 2009; 17(6): 456-463.

46. Cho A, Kwak S, Lee J. Identifying attentional bias and emotional response after appearance-related stimuli exposure. Cyberpsychol Behav Soc Netw. 2013; 16(1): 50-55.

47. Kwak SM, Na DL, Kim G, Kim GS, Lee JH. Use of eye movement to measure smokers' attentional bias to smoking-related cues. Cyberpsychol Behav. 2007; 10(2): 299-304.

48. Kang O, Chang D, Jahng G, et al. Individual differences in smokingrelated cue reactivity in smokers: an eye-tracking and fMRI study. Prog Neuropsychopharmacol Biol Psychiatry. 2012; 38(2): 285-293.

49. Liu XH, Qian MY, Zhou XL, Wang AM. Repeating the stimulus exposure to investigate what happens after initial selective attention to threatening pictures. Personality and Individual Differences. 2006; 40(5): 1007-1016.

50. Yan X, Jiang Y, Wang J, et al. Preconscious attentional bias in cigarette smokers: a probe into awareness modulation on attentional bias. Addict Biol. 2009; 14(4): 478-488.

51. Cox WM, Klinger E. Measuring motivation: the Motivational Structure Questionnaire and Personal Concerns Inventory and their variants. In: Cox WM, Klinger E, eds. Handbook of Motivational Counseling: Goal-Based Approaches to Assessment and Intervention with Addiction and Other Problems. Chichester, UK: WileyBlackwell; 2011: 159-204.

52. Klinger E, Cox WM. The Motivational Structure Questionnaire and Personal Concerns Inventory: psychometric properties. In: Cox WM, Klinger E, eds. Handbook of Motivational Counseling: GoalBased Approaches to Assessment and Intervention with Addiction and Other Problems. Chichester, UK: Wiley-Blackwell; 2011: 177-197.

53. Calitri R, Pothos EM, Tapper K, Brunstrom JM, Rogers PJ. Cognitive Biases to Healthy and Unhealthy Food Words Predict Change in BMI. Obesity (Silver Spring). 2010; 18(12): 2282-2287.

54. Houben K, Roefs A, Jansen A. Guilty pleasures: implicit preferences for high calorie food in restrained eating. Appetite. 2010; 55(1): 18-24.

55. Kemps E, Tiggemann M. Attentional bias for craving-related (chocolate) food cues. Exp Clin Psychopharmacol. 2009; 17(6): 425-433.

56. Tapper K, Pothos EM, Fadardi JS, Ziori E. Restraint, disinhibition and food-related processing bias. Appetite. 2008; 51(2): 335-338.

57. Fadardi J, Bazzaz M, Yazdi A, Nematy M. The role of attentional bias and eating styles in dieters [translated from Persian]. Journal of Clinical Psychology [in Persian]. 2013; 5(17): 57-68.

58. Field M, Eastwood B. Experimental manipulation of attentional bias increases the motivation to drink alcohol. Psychopharmacology (Berl). 2005; 183(3): 350-357.

59. Schoenmakers TM, de Bruin M, Lux IF, et al. Clinical effectiveness of attentional bias modification training in abstinent alcoholic patients. Drug Alcohol Depend. 2010; 109(1-3): 30-36.

60. Cepeda N, Pashler H, Vul E, Wixted J, Rohrer D. Distributed practice in verbal recall tasks: a review and quantitative synthesis. Psychol Bull. 2006; 132(3): 354-380.

61. Greene R. Repetition and spacing effects. In: Roediger HL III, ed. Learning and Memory: A Comprehensive Reference, Vol 2: Cognitive Psychology of Memory. Oxford, UK: Elsevier; 2008: 65-78.

62. Kirchner W. Age differences in short-term retention of rapidly changing information. J Exp Psychol. 1958; 55(4): 352-358.

63. Jaeggi S, Buschkuehl M, Jonides J, Perrig W. Improving fluid intelligence with training on working memory. Proc Natl Acad Sci $U S$ A. 2008; 105(19): 6829-6833. 
64. MacLeod C, Mathews A, Tata P. Attentional bias in emotional disorders. J Abnorm Psychol. 1986; 95(1): 15-20.

65. Bar-Haim Y, Dominique L, Pergamin L, Bakermans-Kranenburg M, van IJzendoorn M. Threat-related attentional bias in anxious and nonanxious individuals: a meta-analytic study. Psychol Bull. 2007; 133(1): 1-24.

66. Bar-Haim Y. Research Review: Attention bias modification (ABM): a novel treatment for anxiety disorders. J Child Psychol Psychiatry. 2010; 51(8): 859-870.
67. Miller WR, Tonigan JS, Longabaugh R. eds. The Drinker Inventory of Consequences (DrInC): An Instrument for Assessing Adverse Consequences of Alcohol Abuse: Test Manual. Washington, DC: US Government Printing Office; 1995.

NIAAA Project MATCH Monograph Series. Vol. 4. NIH Pub. No. 95-3911.

68. Cox WM, Hogan LM, Kristian MR, Race JH. Alcohol attentional bias as a predictor of alcohol abusers' treatment outcome. Drug Alcohol Depend. 2002; 68(3): 237-243. 